$\xi=-1$

\title{
DietSkan: Food Volume Estimation for Dietary Intake Analysis Using 3D Mesh Scanning
}

\author{
Sep Makhsous, Jack Gentsch, Joshua Rollins, Zachary Feingold, and Alexander Mamishev \\ Sensors, Energy, and Automation Laboratory, Department of Electrical Engineering, \\ University of Washington, Seattle WA, USA \\ *Corresponding author E-mail: mamishev@uw.edu
}

\begin{abstract}
The prevalence of obesity, found in more than $38 \%$ of worldwide adults, is causing dietary measurements to become increasingly important. Most methods for tracking dietary intake utilize estimating the amount of food consumed to determine calories and nutritional content. Currently used methods of dietary tracking are either tedious or inaccurate. Our proposed method for dietary tracking is called DietSkan. It combines an off the shelf 3-Dimensional (3D) scanner, the Structure Sensor, with a smartphone application to produce a 3D reconstructed mesh scan of food items. The DietSkan process requires the desired food item to be scanned and exported for volume calculation. Then, using a 3D mesh manipulation tool, a 3D mesh, enclosing the consumed food, is constructed to obtain volume. The volume measurements achieved using the DietSkan algorithm average only 6\% error and allow a user to track their dietary intake simply and effectively. The DietSkan system simplifies the estimation process and improves measurement accuracy when compared to current common practices.
\end{abstract}

Keywords: dietary measurement, $3 D$ mech analysis, volume estimation, and $3 D$ reconstruction.

\section{Introduction}

Dietary tracking is growing increasingly necessary as the rate of obesity has risen over time to about $37.7 \%$ of adults in recent years. The number of obese people worldwide has doubled since 1980; over 600 million people were obese in 2014, and this number has risen since (Yanovski, 2018). In addition to using dietary tracking to combat health issues tied to obesity, accurate dietary tracking helps doctors treating issues like diabetes, where the amount of insulin prescribed is based on food consumption. The demand for accurate measurements within the field of nutrition and diet exists, but the means to track dietary intake systematically and accurately is limited. Several issues exist with current dietary tracking systems, one of which is food diaries where users are burdened to estimate their food consumption, which is often inaccurate due to errors in user estimates of the amount of food consumed (Monteiro \& Cannon, 2015).

Common methodology used with current dietary measurement solutions involve using a visual sensor (camera); these instruments are categorized as Visual Based Measurement (VBM) systems (Shirmohammadi \& Ferrero, 2014). This paper outlines the DietSkan, a smartphone application, which contains a VBM system that quickly and accurately allows a user to estimate his dietary intake. The VBM system used for DietSkan includes a smartphone, and a 3D scanner developed by Occipital, called Structure Sensor (Occipital, 2018). The scanning process takes an average of 5 seconds for one plate with multiple food items and guides the user to move the smartphone around the plate using pop up messages to capture a multi-view 3D reconstructions of the plate. By following this scanning process, the meshes are then post-processed to isolate each food item through a manual segmentation process.
Unlike many VBM systems, this system does not currently contain an automatic segmentation process to isolate the desired food objects and needs to be performed manually. After isolating each item, holes in the mesh are filled, allowing for a simple volume calculation. The 3D reconstructed scans are then processed on the DietSkan database to provide accurate volume estimates for further analysis. Using this volumetric estimation, the nutritional intake of the food eaten can be determined from Nutrition Data System for Research (NDSR) database. This provides users with dietary tracking data to support their nutritional health. The DietSkan system was developed to accurately supply a user with the volume of a food item, which can be further extended to the nutritional content of the consumed food. The overall flowchart of the DietSkan system is shown in Figure 1.

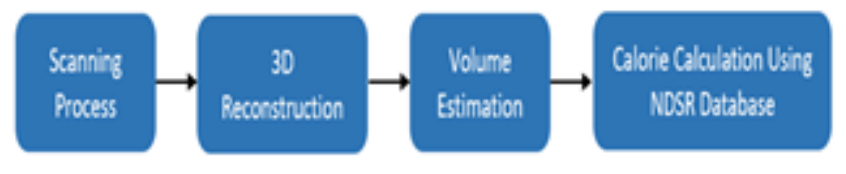

Figure 1. The overall flowchart of the DietSkan system from the scanning process to measuring calorie intake.

The focus of this paper is to provide an overview of the DietSkan system, as well as outlining future efforts with the dietary measurement system. The DietSkan overview includes testing the system individually with single and multi-food plates to measure the accuracy of the system. Then the DietSkan is evaluated and compared to two common methods commonly used for research application and consumer application. The 24-hour logs (24HR) using food frequency questionnaires (FFQ) is commonly used in for research applications, and MyFitnessPal is most commonly used consumer applications) (Pais, Parry, Petrova, \& Rowan, 2018; 
Primer, 2018).

\section{Literature Review}

DietSkan is not the only solution that uses 3D reconstruction to measure dietary intake, in reality, there are multiple approaches in the field of dietary intake using 3D reconstruction and volume estimation. Of these systems, the approach taken by the team at the University of Ottawa is notable for calculating caloric intake through processing of an uploaded 2D image (Pouladzadeh, 2017; Pouladzadeh \& Shirmohammadi, 2017; Pouladzadeh, Shirmohammadi, Bakirov, Bulut, \& Yassine, 2015). The food in the image is divided into food items, and image processing attempts to identify the food items on the plate by using Convolutional Neural Network (CNN). By comparing the portions on the plate to different types of food and questioning the user for confirmation, the food on the plate is identified. The volume is then estimated using a known visual reference required in the 2D image, and each type of food is analyzed with a Support Vector Machine (SVM) method. The results of SVM analysis are then compared with a nutrition database to retrieve caloric information, and the data is stored. Another advanced and commonly used approach, developed by a team at Purdue University, uses a colored index card to capture true dimensions. This method is widely used in multiple nutritional studies. The index card is a 2-dimensional (2D) reference point, which disables the algorithm from capturing depth and texture for accurate volume measurements. In addition to the index card, to calculate the volume estimation of the food, this method uses a single image of the meal while factoring in the geometric properties using shape templates to reconstruct the 3D map of the food. DietCam, developed by a team at Michigan Technical University, uses a similar approach as the index card (Kong, He, Raynor, \& Tan, 2015; Kong \& Tan, 2012). DietCam uses a smartphone camera to calculate the calorie intake and contains image manager, food classifier, and volume estimator, which uses the $3 \mathrm{D}$ reconstruction of the food to calculate the volume. This method is very similar to the DietSkan method, which is presented in this paper, especially the multi-view feature, which allows the user to take three images from all around the plate to capture all the reference points. However, the DietCam method uses an external reference point and requires the user to calibrate the smartphone camera before the scan. The external reference object is a credit card, which allows the algorithm to calculate the actual dimensions of the meal. Use of a credit card limits the algorithm to capture an accurate calculation of the volume, which is satisfactory for general use but it would not be very useful for users such as diabetic patients since the results affect the insulin prescription directly. DietSkan attempts to increase the accuracy and to simplify the process to be used in real life scenarios while producing rapid nutritional estimation. Also, DietSkan focuses on producing higher accuracy information for patients such as people with diabetes to measure the exact dietary intake for consistent prescription of insulin. This is where the DietSkan system proves to be beneficial. Using a Structure Sensor from Occipital Inc. allows for complete scanning of the food, leading to accurate measurements of the food from every angle for full nutritional analysis.

\section{System Overview}

\subsection{DietSkan Application Scanning Algorithm}

The DietSkan application itself utilizes the smartphone camera with Structure Sensor 3D scanner, to produce 3D reconstructed mesh scans of plates with various food items. In a process common in structured light scanning systems including VBMs, an infrared laser, and diffraction grating are coupled to produce a laser grid. This grid is emitted from the structure sensor and is reflected off the food items being scanned. By analyzing the reflected light, the sensor can generate depth data, and thus create a point cloud for fairly accurate volume estimations. Essentially, the
DietSkan application combines 2D data captured by the smartphone camera with depth information from the sensor for a complete triangular 3D mesh. The mesh scan is then postprocessed in a separate application for volume estimation.

\subsection{Scanning Methodology}

The primary goal of the DietSkan system is to quickly and accurately determine the volume of various food items and therefore obtain the nutritional content of food. The scanning methodology for obtaining ideal 3D scans is to capture the scene from all angles and capture the item perfectly along the edges on the bottom surface. During the scanning process, the iPad screen will display the 3D scan as it is generated. Once the food has been scanned thoroughly, and the desired object is completely covered, the scanning can be stopped, and the mesh is saved to an attached Dropbox account.

At this point, the linked Dropbox account contains the 3D mesh scan in the form of an object file containing the expansive series of vertices that comprise the triangular mesh, and a preview JPEG image taken by the camera during the scanning process. The volume is then calculated using 3D mesh manipulation tools to postprocess the food item reconstruction. The process of scanning and uploading an object using the DietSkan application takes an average of one minute.

\subsection{Post-Processing the Mesh Scans}

Post-processing of the scans was done using MeshMixer, a free 3D reconstruction tool (Autodesk, 2017). This application was chosen over several other tools due to its simplicity to segment individual items in the scan, stability to run and fill all holes in the scan and perform the volume calculation with high accuracy. Other additional tools were tested to find the most efficient, but most could not close the holes in the mesh, were costly, or, most importantly, could not calculate the volume of a watertight mesh. Aside from free tools.

Within MeshMixer, food items can be manually selected, and extraneous portions of the mesh (i.e. the plate and food items that are not of interest) are deleted. The 3D mesh scan of the food item of interest must then be made watertight, contain no holes, to achieve an accurate volume estimation. Once the scan is made watertight, MeshMixer quickly calculates the volume of the object The post-processing time is typically between one to three minutes per food item.

Initial testing on real foods were performed at Harborview Medical Center, Seattle, Washington, United States; and the scans were largely inaccurate due to regions near the plate being filled with holes. Further experimentation with plastic food items allowed the team to practice taking scans, and eventually consistently scan entire meals with ease.

\subsection{Processed Food Scan Results}

The results compiled from twenty-four scans of plastic foods show a mean error of only $6 \%$ overall. The average error for single food items on a plate was $5.3 \%$, for multiple food items on a plate not touching (MS) the average was 7.4\%, and for multiple items on a plate touching (MT) the average was $5.0 \%$. The most inaccurate scans consisted of multiple items, with $16 \%$ error for MS3_Apple, $15 \%$ error for MS4_Apple, and 14\% error for MS2_Croissant. The percent differences for each scan is shown in Figure 9. With the small percentage difference in volume measurements for each food item, the variance in measurements was very small - with most items falling between an average standard deviation of $4 \%$ error, indicating the post-processed results were very consistent in general. Thus, by taking slow scans of food items and carefully segmenting the meshes by hand, very accurate measurements of the food volume can be obtained.

Due to the high accuracy of the scanning procedure, the primary source of error can be attributed to an incomplete scan of the ob- 
ject or errors in separating food items from the plate. The underside of the apple is estimated by MeshMixer's hole-filling algorithm and is hence overestimated. The underside of an apple should have a curved surface with a concave curvature to the core at the center of the apple, but MeshMixer is unable to predict this due to the scan's missing information. Despite the estimation necessary, when the mesh interfaces with the plate, the apple had a percentage error of 5\%, and the croissant an error of $14 \%$ for the scan that had the apple and croissant touching, MT2. This error likely arose from the interface between the apple and croissant, where the two meshes are separated to ensure their volumes can be calculated.

Thus, we attribute our errors in volume calculation to the algorithm's inability to consistently scan the entirety of each food object, in particular, the intersection region between food items and the plate. Sections of each scan that are viewable from all angles, on the contrary, produce ideal scans and are thus 3D reconstructed with ease.

\section{Dietskan Comparison Study}

An experiment was conducted with the goal of comparing the DietSkan scanner accuracy to other dietary tracking methods common in the market. Participants volunteered to consume a prepared plate of food and utilize a variety of dietary tracking methods to measure their nutritional intake. Users were split into groups with varying amounts of information given about their meal. One group was served a plate with no information, others were given a list of recipes to pick one they considered most accurate, and a third group was given the exact recipe for the prepared food. The DietSkan app and structure sensor were used to take mesh scans of a plate of food. Users were also directed to a related survey where users estimated the volume of the main ingredients were consumed. Control of the volume measurements consisted of the research team hand measuring the mass of each food item in grams before and after serving the food to participants. Other methods utilized to estimate intake included a 24-hour dietary recall called the Automated Self-Administered 24-Hour (ASA24) survey, and a consumer iPhone application used to track dietary intake named MyFitnessPal. The DietSkan app method requires the user to scan the food before they eat, and after if leftovers remain. Each dietary tracking method requires users only to estimate the amount of each ingredient that was consumed. Afterward, the collected 3D meshes are converted to volumes in MeshMixer, a 3D mesh processing software. For all of the surveys, the participants each estimated their intake values and submitted their surveys to an online database for the collection. The scanned volume and surveyed volumes are then converted into various nutritional data for each participant, including but not limited to calories, fat, sodium, sugar, protein, and carbs.

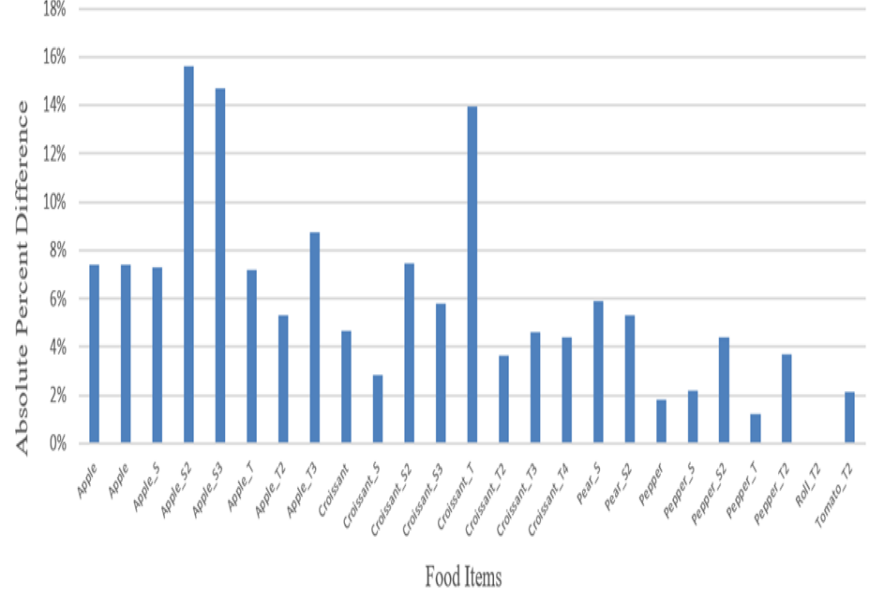

Figure 4. Error estimation analysis illustrates the complexity introduced by non-separated foods. The '_S' indicates the item was separated from other food items. The '_T' suffix indicates the items on the plate were touching.

\subsection{Analysis of Results}

Figure 5 shows the absolute percentage error calculated between the measured carbohydrate and calculated value while comparing the results between the three methods used; the DietSkan application, 24HR recall, and MyFitnessPal. The DietSkan method is drastically underestimating the nutritional information for all participants, with some participants achieving nearly no calculated nutritional intake. Self-reporting methods, including the online survey, 24-hour recall, and iPhone application MyFitnessPal, will occasionally have drastically inaccurate results but have a low percent error on the average. The average percent error for the DietSkan Scanner is $-78.64 \%$, $27.99 \%$ for the survey, $61.84 \%$ for 24-hour recall, and 32.19\% for MyFitnessPal.

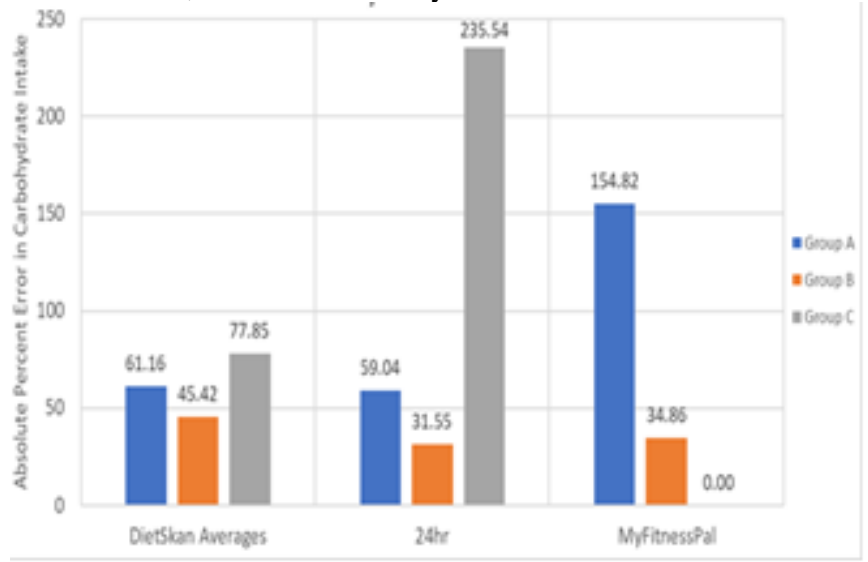

Figure 5. The absolute percentage error calculated between the measured carbohydrate and the calculated value is presented while comparing the results of the three methods used.

\section{Conclusions and Future Work}

The DietSkan application for obtaining accurate volumetric estimations through $3 \mathrm{D}$ reconstruction of a scanned object is highly useful to the field of dietary tracking. The fact that it is simple to use, while maintaining an average of $94 \%$ accuracy and consuming little time improves the quality of dietary tracking, allowing a variety of users to implement DietSkan for various use cases without having to sacrifice time or money. Due to the current limitations with post-processing scans that were not scanned well at the resting surfaces edges, future work to improve the DietSkan system will include an automated scanning system that is careful to maintain all boundaries in the scans. This would improve the consistency of the scans and increase the reliability of post-processing, hole-filling, and thus volume estimations. From this, the system would primarily benefit from reliably scanning the bottom edges of the food as well, to minimize lost accuracy due to the MeshMixer hole filling limitations. Finding the means to automate the segmentation process, ranging from standardizing scanning techniques and developing algorithms to separate food from plate automatically, would greatly benefit the accuracy and usability of the DietSkan system.

Although the DietSkan system achieves highly accurate results with an average of only $6 \%$ error, there is plenty of room for improvement to the system. The DietSkan system is intended to be improved through automating the means to segment food items from plates, moving from the automatic removal of plates to automatically separating noticeably different food items. From here, scans can be automatically segmented and processed once the user has uploaded them to Dropbox, which would drastically reduce the processing time. This automated use case will be developed around an automated scanning hardware system, which is currently being designed and documentation is in the publication process. For the DietSkan system's commercial use, thorough scanning must be properly instructed. User tests for the DietSkan system have insofar failed, as users were unable to produce scans that properly capture edges of the food. This is a combination of a poor 
user instruction and the user's haste in taking a scan. Future systems will be carefully tested and deployed as to capture these edges, increasing the chances that output scans will have easily closable volumes. Overall, further increasing the accuracy of holefilling to reduce the likelihood of removing large volumes from food items, as well as automating the food and plate segmentation and scanning processes are the focus of the future development of DietSkan.

\section{References}

[1] Kong, Fanyu, He, Hongsheng, Raynor, Hollie A., \& Tan, Jindong (2015). DietCam: Multi-view regular shape food recognition with a camera phone. Pervasive and Mobile Computing, 19, 108-121. doi:https://doi.org/10.1016/j.pmcj.2014.05.012

[2] Kong, Fanyu, \& Tan, Jindong. (2012). DietCam: Automatic dietary assessment with mobile camera phones. Pervasive and Mobile Computing, 8(1), 147-163. doi:https://doi.org/10.1016/j.pmcj.2011.07.003

[3] Monteiro, Carlos A., \& Cannon, Geoffrey. (2015). Calories do not add up. Public Health Nutrition; Cambridge, 18(4), 569-570. doi:http://dx.doi.org/10.1017/S1368980015000014

[4] Occipital. (2018). Structure Sensor 3D Scanner. Retrieved from https://occipital.com/

[5] Pais, S, Parry, D, Petrova, K, \& Rowan, J. (2018). Acceptance of Using an Ecosystem of Mobile Apps for Use in Diabetes Clinic for Self-Management of Gestational Diabetes Mellitus. Paper presented at the MEDINFO 2017.

[6] Pouladzadeh, Parisa. (2017). A Cloud-Assisted Mobile Food Recognition System. Université d'Ottawa/University of Ottawa

[7] Pouladzadeh, Parisa, \& Shirmohammadi, Shervin. (2017). Mobile multi-food recognition using deep learning. ACM Transactions on Multimedia Computing, Communications, and Applications (TOMM), 13(3s), 36

[8] Pouladzadeh, Parisa, Shirmohammadi, Shervin, Bakirov, Aslan, Bulut, Ahmet, \& Yassine, Abdulsalam. (2015). Cloud-based SVM for food categorization. Multimedia Tools and Applications, 74(14), 5243-5260. doi:10.1007/s11042-014-2116-x

[9] Primer, Dietary Assessment. (2018, 2016/10/19/21:24:24). 24-hour Dietary Recall (24HR) At a Glance. Retrieved from https://dietassessmentprimer.cancer.gov/profiles/recall/

[10] Shirmohammadi, S., \& Ferrero, A. (2014). Camera as the instrument: the rising trend of vision based measurement. IEEE Instrumentation \& Measurement Magazine, 17(3), 41-47. doi:10.1109/MIM.2014.6825388

[11] Yanovski, Jack A. (2018). Obesity: Trends in underweight and obesity-scale of the problem. Nature Reviews Endocrinology, 14(1), 